\title{
Quatro espécies novas de Tropicanus DeLong (Hemiptera, Cicadellidae) ${ }^{1}$
}

\author{
Keti M. R. Zanol ${ }^{2}$ \\ ${ }^{1}$ Contribuição número 1635 do Departamento de Zoologia, Universidade Federal do Paraná. \\ 2 Departamento de Zoologia, Universidade Federal do Paraná. Caixa Postal 19020, 81531-980 Curitiba, Paraná, Brasil. \\ Bolsista do CNPq. E-mail: kzanol@ufpr.br
}

\begin{abstract}
Four new species of Tropicanus DeLong (Hemiptera: Cicadellidae). Four new species of Tropicanus are described in the subgenus Tropicanus: T. taulipangus sp. nov., T. amazonicus sp. nov., T. chapadensis sp. nov. and T. lisei sp. nov., all from Brazil.

KEY WORDS. Deltocephalinae; Neotropical region; taxonomy.
\end{abstract}

RESUMO. Quatro espécies novas de Tropicanus DeLong (Hemiptera: Cicadellidae). São descritas quatro novas espécies no subgênero Tropicanus: T. taulipangus sp. nov., T. amazonicus sp. nov., T. chapadensis sp. nov. e T. lisei sp. nov., todas para o Brasil.

PALAVRAS-CHAVE. Deltocephalinae; região Neotropical; taxonomia.

Tropicanus foi descrito por DELong (1944) como subgênero de Phlepsius designando como espécie-tipo Allygus costomaculatus Van Duzee e descreveu três espécies. DeLong \& KNuLL (1946) consideraram Tropicanus como gênero. OMAN (1949) listou as espécies neárticas transferindo para o gênero Phlepsius pulchripennis Baker. Linnavuori (1959) transferiu Phlepsius annulatus para Tropicanus e descreveu T. bicornis; neste mesmo trabalho descreveu o subgênero Cabimanus designando como espécie-tipo, Phlepsius singularis. DeLong. ZANoL (1991) descreveu quatro espécies ficando o gênero composto por 12 espécies. Neste trabalho são descritas quatro novas espécies no subgênero Tropicanus: Tropicanus taulipangus sp. nov., Tropicanus amazonicus sp. nov., Tropicanus chapadensis sp. nov., Tropicanus lisei sp. nov., todas para o Brasil.

As espécies de Tropicanus caracterizam-se por apresentar os ocel os na margem anterior da cabeça, visíveis de cima. Sutura coronal atingindo a metade da coroa. Genas sinuosas. Anteclípeo de lados paral elos. Pronoto tão largo quanto a cabeça. Asas anteriores semitransparentes, com fal sas veias e com três células anteapicais fechadas; a externa com extranumerária; clavo com uma veia extranumerária entre as veias anais e uma veia extranumerária entre $1 \mathrm{~A}$ e a sutura claval. Espinulação dos fêmures posteriores $2+2+1$ e das tíbias anteriores $1+4$ (subgênero Tropicanus) ou $3+4$ (subgênero Cabimanus). Pigóforo com ápice arredondado. Valva genital triangular. Placas subgenitais triangulares com a metade apical mais estreita pouco esclerotinizada e corrugada; macrocerdas unisseriadas. Conetivo em forma de " $Y$ " invertido. Edeago assimétrico; gonóporo na superfície ventral.

O material está depositado no Instituto de Pesquisa da Amazônia, Manaus, Amazonas (INPA); Museu Paraense Emilio Goeldi, Belém, Pará (MEGP) e Coleção de Entomologia Pe J. S.
Moure, Departamento de Zoologia, UFPR, Curitiba, Paraná (DZUP).

\section{Elenco das espécies}

Tropicanus (Tropicanus) DeLong, 1944 Phlepsius (Tropicanus) DeLong, 1944: 87.

Tropicanus; DeLong \& Knull, 1946: 54.

Tropicanus (Tropicanus) costomaculatus (Van Duzee, 1894)

Allygus costomaculatus Van Duzee, 1894: 207.

[Eutettix] costomaculata; Ball, 1901: 48.

Phlepsius (Dixianus) costomaculatus; Ball, 1918: 388.

Phlepsius (Tropicanus) costomaculatus; DeLong, 1944: 89.

Tropicanus costomaculatus; DeLong \& Knull, 1946: 54.

Tropicanus (Tropicanus) costomaculatus; Linnavuori, 1959: 202.

Distribuição: Estados Unidos da América, M éxico, Guatemala, Brasil.

Tropicanus (Tropicanus) pulchripennis (Baker, 1898)

Phlepsius pulchripennis Baker, 1898: 65.

Tropicanus pulchripennis; Oman, 1949: 144.

Distribuicão: Estados Unidos da América.

Tropicanus (Tropicanus) calidus (DeLong, 1944)

Phlepsius calidus DeLong, 1944: 90.

Tropicanus (Tropicanus) calidus; Linnavuori, 1959: 203.

Tropicanus calidus; Metcalf, 1967: 569.

Distribuição: México, Guatemala.

Tropicanus (Tropicanus) digitus (DeLong, 1944)

Phlepsius digitus DeLong, 1944: 89.

Tropicanus digitus; Metcalf, 1967: 571.

Distribuição: México. 
Tropicanus (Tropicanus) flectus (DeLong, 1944)

Phlepsius flectus DeLong, 1944: 88-89.

Tropicanus (Tropicanus) flectus; Linnavuori, 1959: 203.

Tropicanus flectus; Metcalf, 1967: 571.

Distribuição: México, Nicarágua, Panamá.

Tropicanus (Tropicanus) annulatus (Osborn, 1923)

Phlepsius annulatus Osborn, 1923: 61-62.

Tropicanus (Tropicanus) annulatus; Linnavuori, 1959: 203.

Distribuição: Brasil.

Tropicanus (Tropicanus) bicornis Linnavuori, 1959

Tropicanus (Tropicanus) bicornis Linnavuori, 1959: 203.

Distribuição: Argentina.

Tropicanus (Tropicanus) chiapasus Zanol, 1991

Tropicanus (Tropicanus) chiapasus Zanol, 1991: 82.

Distribuição: México.

Tropicanus (Tropicanus) irroratus Zanol, 1991

Tropicanus (Tropicanus) irroratus Zanol, 1991: 79.

Distribuição: México.

Tropicanus (Tropicanus) mexicanus Zanol, 1991

Tropicanus (Tropicanus) mexicanus Zanol, 1991: 82.

Distribuição: México.

Tropicanus (Tropicanus) punctatus (Osborn, 1923)

Eutettix punctatus Osborn, 1923: 50.

Menosoma punctata; Linnavuori, 1959: 185.

Tropicanus (Tropicanus) punctatus; Linnavuori \& DeLong, 1977: 561.

Distribuição: Bolívia.

Tropicanus (Cabimanus) Linnavuori, 1959

Tropicanus (Cabimanus) Linnavuori, 1959: 204

Tropicanus (Cabimanus) singularis (DeLong, 1944)

Phlepsius singularis DeLong, 1944: 92.

Tropicanus (Cabimanus) singularis; Linnavuori, 1959: 204.

Tropicanus singularis; Metcalf, 1967: 572.

Distribuição: México, Panamá.

\section{Tropicanus (Tropicanus) taulipangus sp. nov. Figs 1-7}

Cabeça. Coloração marfim; coroa com quatro manchas na margem anterior; duas manchas marrom-escuras, junto aos ocel os e duas manchas marrom-claras, no meio; sutura coronal com uma faixa longitudinal que diverge apicalmente formando dois braços que se projetam para trás, sobre o disco, numa mancha ovalada, marrom-clara. Face marfim com manchas dispersas e irregulares marrons. Pronoto amarelado com manchas marrom-claras. Asas anteriores amarelas com manchas e pontuações marrom-escuras; venação marrom.

Pigóforo com a margem ventral voltada para dentro, de forma mais ou menos triangular. Margem interna das placas subgenitais com um processo dentiforme, interno, esclerotinizado, junto ao estreitamento. Estilos digitiformes; apófises com textura imbricada. Edeago com três apêndices saindo junto ao gonóporo; um apêndice apical dorsalmente curvo para trás; dois apêndices subapicais, ventrais, voltados para trás, um de cada lado do gonóporo; o apêndice da esquerda mais ou menos triangular voltado para trás e para dentro e, o apêndice da direita maior, sinuoso e voltado para trás.

Fêmea. Desconhecida.

Comprimento. Macho 4,50 mm.

Tipo e localidade-tipo. Holótipo macho. BrasIL: Roraima, Rio Uraricoera, Il ha de Maracá. J.A. Rafael, E. Brasil eL.S. Aquino leg. (INPA).

Etimologia. Tupi - Taulipang, nome de tribo caribe habitante do rio Uraricoera.

\section{Tropicanus (Tropicanus) amazonicus sp. nov. Figs 8-16}

Cabeça. Coloração marfim; coroa com quatro manchas na margem anterior, duas próximas aos ocelos, marrom-escuras e duas medianas marrom-claras; sutura coronal com uma faixa longitudinal que diverge apical mente formando dois braços que se projetam para trás, sobre o disco, numa mancha, de contorno irregular, marrom-clara. Face amarela com manchas dispersas e irregulares marrom-claras. Pronoto amarelado com manchas marrom-claras. Asas anteriores esbranquiçadas com manchas e pontuações marrom-escuras; venação marrom-clara.

Pigóforo sem apêndices. Estilos com apófises afiladas, levemente curvas para fora. Edeago com três apêndices saindo junto ao gonóporo; um apêndice dorso-apical curvo para trás; dois apêndices subapicais, ventrais, um de cada lado do gonóporo; o apêndice da direita maior, sinuoso, voltado para trás e o apêndice da esquerda levemente curvo para trás e para dentro.

Fêmea. Externamente semelhante ao macho porém mais escura. Sétimo esterno com a margem posterior escavada.

Tipo e localidade-tipo. Holótipo macho. Brasil. Amapá: Curau, 19.XI.1981, L.S. Gorayeb e equipeleg. (DZUP). Parátipos. Ibidem, 4 machos (DZUP, MPEG), 1 fêmea (DZUP). Pará: Monte Alegre, 16-20.I.1992, A. L. Henriques leg., 1 macho, 1 fêmea (DZUP); Santarém (vila Inanu), 26.XII.94-2.II.1995, L. Aquino leg. 1 macho, 1 fêmea (INPA); Bragança-Tracuateua, 2-4.XI.1990, 1 fêmea (INPA). Amazonas: Rodovia BR 319 km 268, 20.I.1978, J. Arias, 1 macho (INPA). Roraima: Rio Uraricoera, Ilha de Maracá, 18-28.VIII.1987, J.A. Rafael, L.S. Aquino, J.F. Vidal e E. Binda leg., 2 machos, 2 fêmeas; Ibidem, 21-30.XI.1987, 2 machos, 1 fêmea; Ibidem, 18-28.VIII.1987, 1 fêmea (INPA, DZUP). Rondônia: Guaporé, 1213'19"S 6032'44"W, 23.I.2006, J.A. Rafael e F.F. Xavier Filho, 1 macho, 1 fêmea (INPA).

Comprimento. Macho 4,00-4,20 mm. Fêmea 4,30-4,60 mm.

Variação intraespecífica. O número de veias extranumerárias na célula anteapical externa pode variar de 1 a 3 veias inclusive quando comparamos a asa direita com a asa esquerda no mesmo indivíduo.

Etimologia. O nome é alusivo à região amazônica onde os espécimens foram coletados.

Revista Brasileira de Zoologia 23 (4): 1251-1255, dezembro 2006 

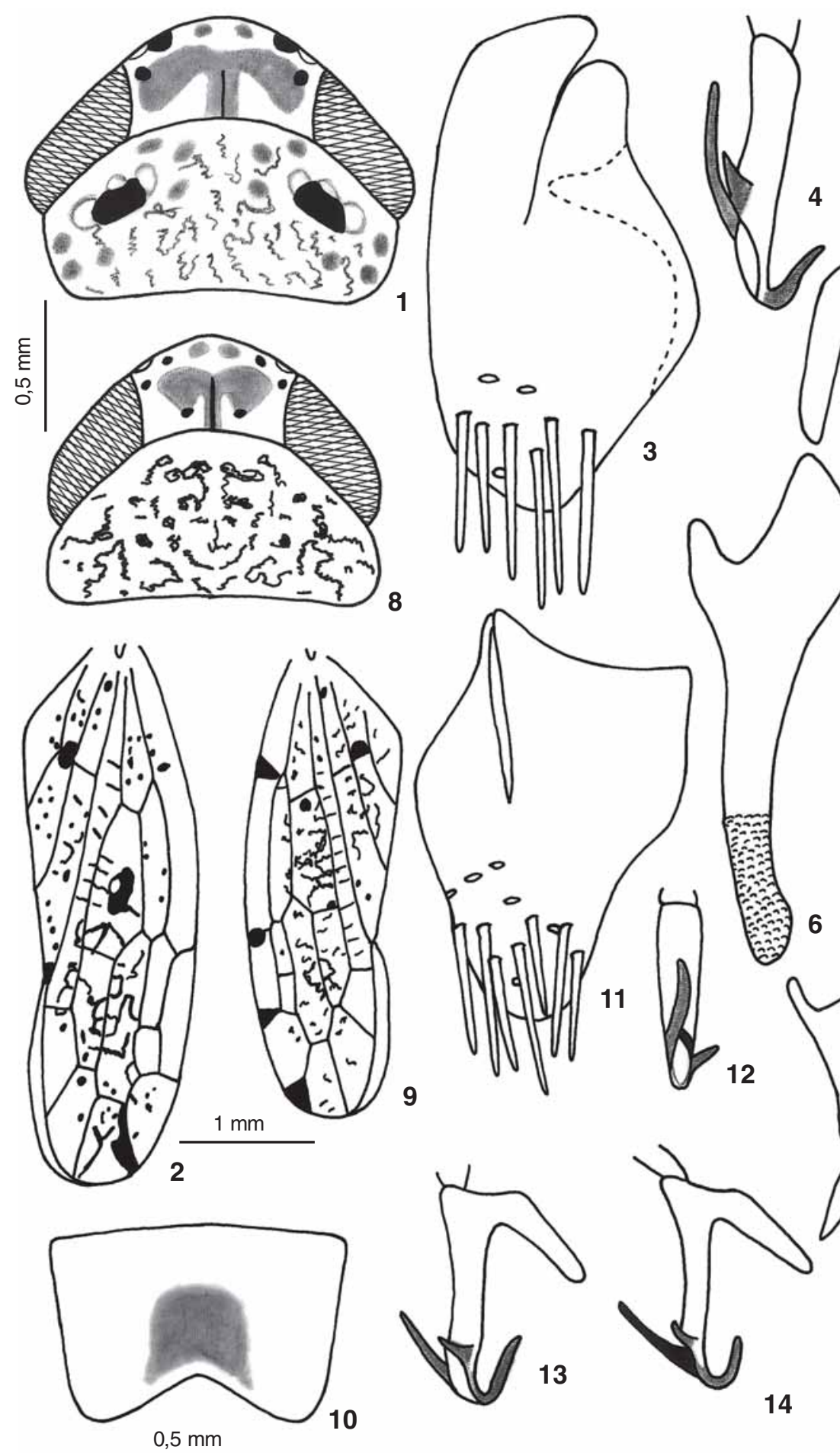

11
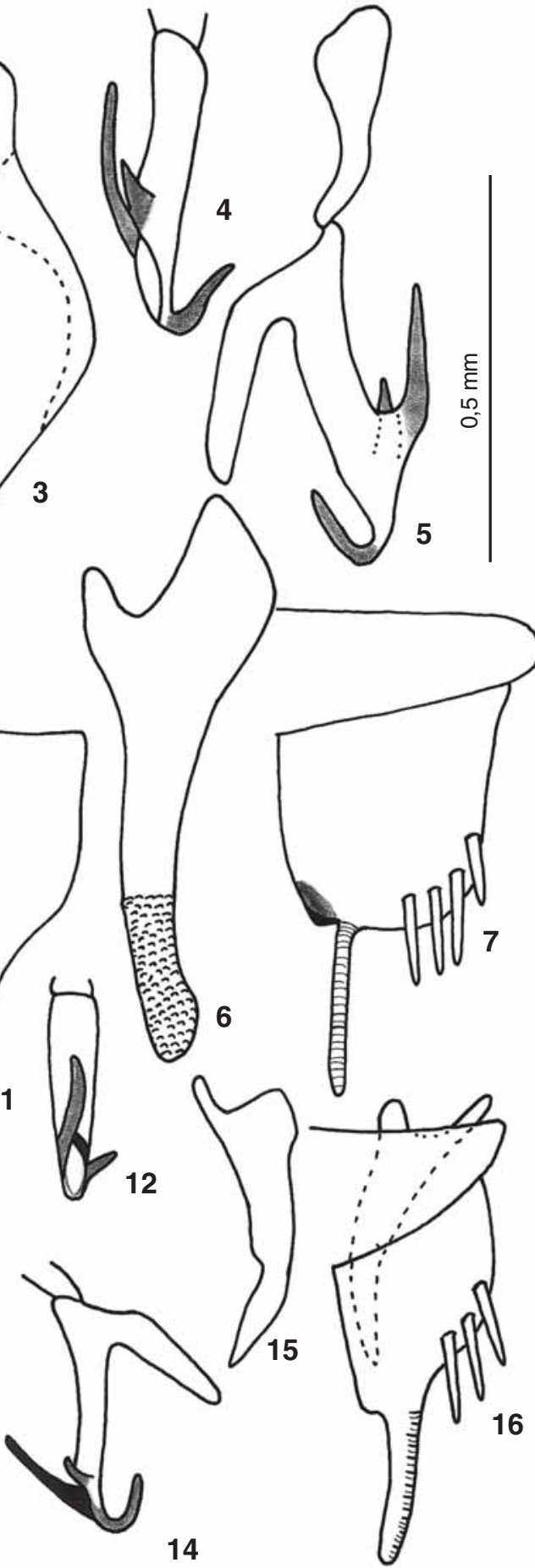

Figs 1-16. (1-7) Tropicanus taulipangus sp. nov.: (1) cabeça e pronoto; (2) asa anterior; (3) pigóforo; (4) edeago, ventro-lateral; (5) conetivo e edeago, lateral; (6) estilo; (7) valva genital e placa subgenital; (8-16) Tropicanus amazonicus sp. nov.: (8) cabeça e pronoto; (9) asa anterior; (10) sétimo esterno da fêmea; (11) pigóforo; (12) edeago, lateral; (13) edeago, ventro-lateral; (14) edeago, lateral; (15) estilo; (16) valva genital, placa subgenital e estilo. 

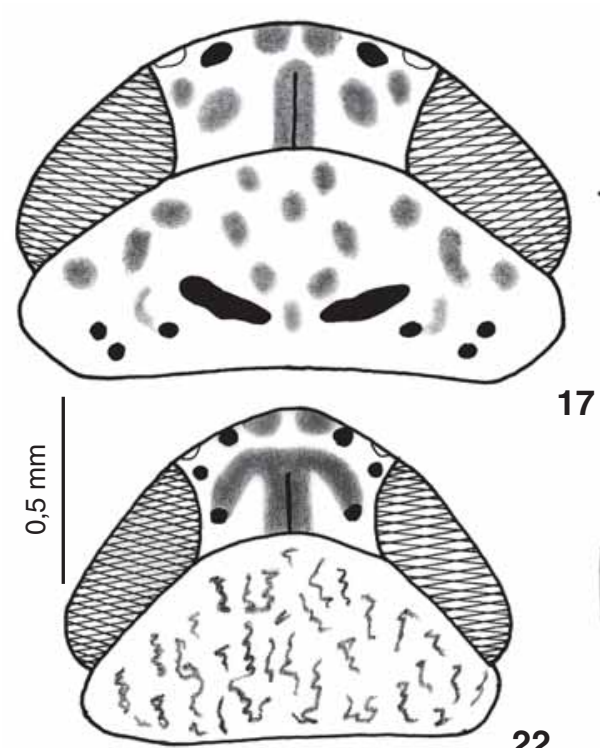

17

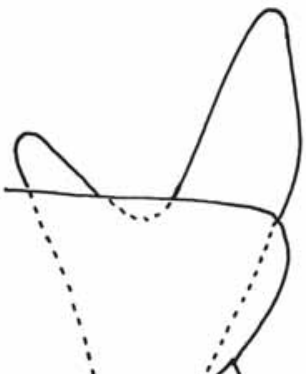

22
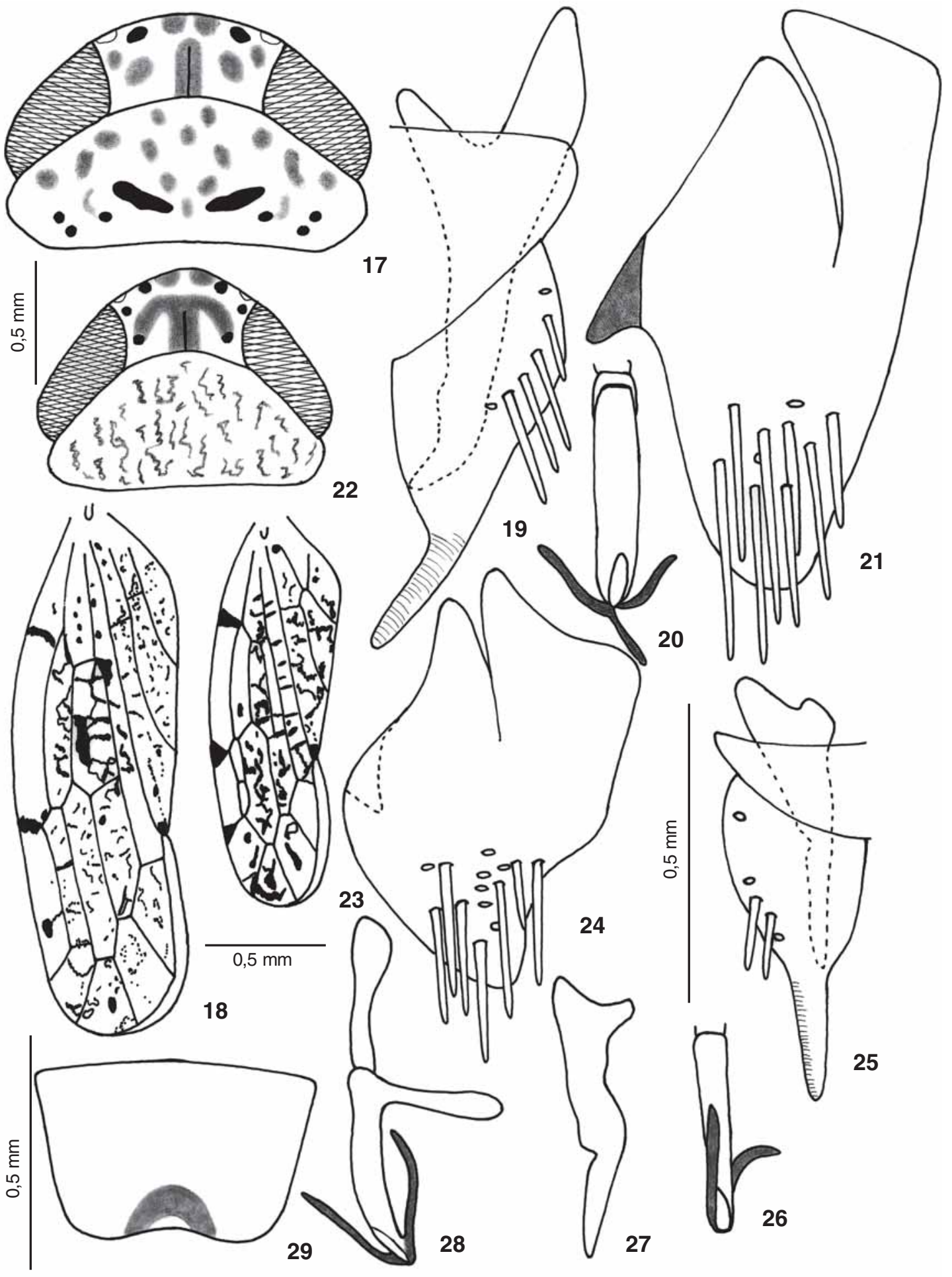

24

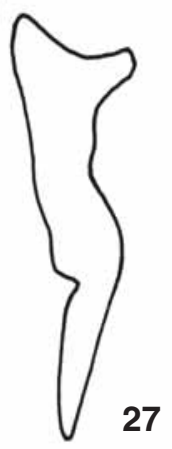

20

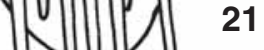

21

25

Figs 17-29. (17-21) Tropicanus chapadensis sp. nov.: (17) cabeça e pronoto; (18) asa anterior; (19) valva genital, placa subgenital e estilo; (20) edeago, ventral; (21) pigóforo; (22-29). Tropicanus lisei sp. nov.: (22) cabeça e pronoto; (23) asa anterior; (24) pigóforo; (25) valva genital, placa subgenital e estilo; (26) edeago, ventral; (27) estilo; (28) conetivo e edeago, lateral; (29) sétimo esterno da fêmea. 


\section{Tropicanus (Tropicanus) chapadensis sp. nov.} Figs 17-21

Cabeça. Coloração amarela; coroa com quatro manchas arredondadas, na margem anterior, marrom-escuras; sutura coronal com uma faixa marrom-clara; região discal com duas manchas marrom-claras, de cada lado da sutura coronal. Face amarela irrorada de marrom. Pronoto amarelado com manchas marrons. Asas anteriores amareladas com manchas e pontuações marrons; venação marrom.

Pigóforo com a margem ventral projetada num processo mais ou menos triangular

Estilos com apófises curvas para dentro. Edeago com dois apêndices dorso-apicais saindo junto ao gonóporo; o apêndice da esquerda sinuoso e voltado para trás e, o apêndice da direita birramoso; o ramo menor, voltado para cima e o ramo maior, sinuoso voltado para trás e para fora

Fêmea. Desconhecida.

Comprimento. Macho 5,10 mm.

Tipo elocalidade-tipo. Holótipo macho. BrASIL: Mato Grosso, Chapada dos Guimarães (Colégio Agrícola Buriti), 8-13.II. 1986, I.S. Gorayeb leg. (DZUP).

Etimologia. O nome é alusivo à localidade onde o espécime foi coletado.

\section{Tropicanus (Tropicanus) lisei sp. nov. Figs 22-29}

Cabeça. Coloração marfim; coroa com cinco manchas marrom-escuras, duas na margem anterior e três sobre disco; sutura coronal com uma faixa longitudinal que diverge apicalmente formando dois braços que se projetam para trás, sobre o disco, numa mancha, de contorno irregular, marrom-clara. Face amarela com manchas e suturas marrom-claras; frontoclípeo com arcos laterais marrons. Pronoto amarelado com manchas marrom-claras. Asas anteriores esbranquiçadas com manchas e pontuações marrom-escuras; venação marrom.

Pigóforo com a margem ventral voltada para dentro de forma mais ou menos triangular. Estilos digitiformes; apófises afiladas. Edeago com dois apêndices saindo junto ao gonóporo; um apêndice dorso-apical sinuoso curvo para trás e para cima e um apêndice ventro-apical sinuoso e voltado para trás.

Fêmea. Externamente semelhante ao macho. Sétimo esterno com a margem posterior sinuosa.

Tipo e localidade-tipo. Holótipo macho. BrASIL: Roraima, Normandia, V.1991, J.A. Rafael, R.A. Rocha eJ. Vidal leg., (INPA). Parátipos. Ibidem, 2 machos, 1 fêmea (INPA, DZUP); Bonfim, Rio Tacutu,.1991, J.A. Rafael, R.A. Rocha e J. Vidal leg., (INPA).
Comprimento. Macho 4,00-4,20 mm. Fêmea 4,60 mm.

Etimologia. O nome é dedicado ao Prof. Dr. Arno Antonio Lise, da Pontifícia Universidade Católica do Rio Grande do Sul.

\section{REFERÊNCIAS BILIOGRÁFICAS}

BAKER, C.F. 1898. Five new specie of Phlepsius. Entomological News, Philadelphia, 9 (3): 65-67.

BALL, E.D. 1901. Some new Jassidae from the rocky Mountain and Pacific region. Canadian Entomologist, Ottawa, 33 (2): 45-51.

BALL, E.D. 1918. The phlepsids of Mexico and Central America (Homoptera, Cicadellidae). Annals of the Entomological Society of America, Lanham, 11 (4): 381-391.

DeLong, D.M. 1944. The Mexican species of Phlepsius (Homoptera: Cicadellidae). Proceedings of the Entomological Society of Washington, Washington, 46 (4): 85-94.

DeLong, D.M. \& R. Knull. 1946. Checklist of the Cicadellidae (Homoptera) of America, North of Mexico. Ohio State University Graduate School Student, Biological Science Series, Columbus, 1: 1-102.

LINNAVUORI, R. 1959. Revision of the Neotropical Deltocephalinae and some related subfamilies. Annales Zoologici Societatis Zoologicae Botanicae Fennicae Vanamo, Helsinki, 20 (1): 1-370.

Linnavuori, R \& D.M. DeLong. 1977. New Deltocephaline leafhoppersfrom Central America (Homoptera: Cicadellidae) and illustations of some Osborn bolivian species. Journal of the Kansas Entomological Society, Lawrence, 50 (4): 558-568.

Metcalf, Z. P. 1967. General Catalogue of the Homoptera. Fascicle VI, Cicadelloidea, Part 10. Euscelidae. Section I. Washington, Agricultural Research Service, United States Departament of Agriculture, 1077p.

OmAn, P.W. 1949. The Nearctic leafhopper (Homoptera: Cicadellidae). A generic Classification and check list. Memoirs of the Entomological Society of Washington, Washington, 3: 1-253.

Osborn, H. 1923. II. Neotropical Homoptera of the Carnegie Museum. Part 2. Records and descriptions of five new genera and sixty-five new species of the subfamily Jassinae. Annals of the Carnegie Museum, Pittsburgh, 25 (1): 27-79.

VAN DuzeE, E. P. 1894. Descriptions of somenew north american homopterous insects. Bulletin Buffalo Society of Natural Science, Buffalo, 5: 205-216.

ZANol, K.M.R. 1991. Descrição de quatro espécies novas de Tropicanus DeLong (Homoptera: Cicadellidae). Revista Brasileira de Zoologia, Curitiba, 7 (1-2): 79-83.

Recebido em 21.VI.2006; aceito em 22.XI.2006. 